good relationship with their partner. Complications were experienced by $123(22.9 \%)$ of women during this pregnancy and commonest was gestational diabetes. The presence of depression was significantly associated with living with extended family $(\mathrm{p}=0.033)$ and in-laws $(\mathrm{p}=0.014)$. Multi parity ( $>2$ children $)$ $(\mathrm{p}=0.008)$, partner's substance use $(\mathrm{p}=0.002)$, inadequate family support $(\mathrm{p}=0.024)$, inadequate partner's support $(\mathrm{p}=0.003)$, unsatisfactory relationship with partner $(\mathrm{p}=0.000)$ and unplanned pregnancies $(p=0.001)$ were also associated with depression. Logistic regression analysis indicated a significant association between depression with partner's substance use, unavailability of family support and poor relationship with the partner.

Conclusion. Around one-third of mothers were having antenatal depression. Several spouse related factors and unsatisfactory family support were associated with depression among antenatal mothers.

\section{An analytical cross-sectional study to describe and compare the mental health status of doctors and medical undergraduates in selected institutions in Colombo, Sri Lanka during COVID-19 pandemic \\ Chathurie Suraweera ${ }^{1 \star}$, Iresha Perera ${ }^{1}$, Priyanka Rupasinghe ${ }^{2}$ and Janith Galhenage ${ }^{1}$ \\ ${ }^{1}$ Professorial Psychiatry Unit, National Hospital of Sri Lanka and ${ }^{2}$ Department of Paediatric Neurology, Lady Ridgeway Hospital for Children, Colombo \\ ${ }^{*}$ Corresponding author.}

doi: 10.1192/bjo.2021.777

Aims. The aim of this study was to describe and compare the mental health status of doctors and medical undergraduates in selected institutions during COVID-19 pandemic.

Method. A cross-sectional analytical study was conducted among doctors working in major tertiary care hospitals two of which, risk is unpredictable and high, the other where all patients are positive for COVID-19 and among medical undergraduates. The doctors were selected using disproportionate stratified sampling and medical undergraduates using stratified cluster sampling. Data were gathered using a Google form containing socio-demographic details, perception on the pandemic and the General Health Questionnaire-12(GHQ-12).

Result. There were 468 participants in the study and among them $243(51.9 \%)$ were doctors. Mean age of the doctors' is 34.54 ( $\mathrm{SD}=$ $7.43)$ years and more than half $(50.06 \%)$ were in post graduate training. Majority were worried about their health (65\%) and their loved one's health (90.1\%). Among doctors 220(90.5\%) felt that they have moderate or higher risk of acquiring COVID-19 and $15.6 \%$ would not have worked due to the risk. According to GHQ-12, 182(74\%) doctors were psychologically distressed (mean GHQ $=12.64, \mathrm{SD}=4.54)$ and it was significantly associated with age less than 35 years $(\mathrm{p}=0.039)$ and worry about interruption of their daily routines $(\mathrm{p}=0.010)$.

The mean age of 225 medical undergraduates was 25.20 $(\mathrm{SD}=1.34)$ years and $176(78.2 \%)$ of the participants were psychologically distressed (mean $\mathrm{GHQ}=14.32, \mathrm{SD}=6.67$ ). Majority $(59.11 \%)$ believed that they are at high risk of getting COVID-19. Their distress was significantly associated with the worry about the impact of COVID-19 related restrictions on their daily routines $(p=0.000)$. Binomial logistic regression confirmed that doctors were distressed due to impact on their income whereas both doctors and medical undergraduates were distressed due to impact on daily routines.
Conclusion. Nearly three quarter of both doctors and medical undergraduates were psychologically distressed during COVID-19 pandemic. The worry was due to contracting illness, financial issues and the COVID-19 regulations.

\section{Prevalence and associations of psychoactive substance use among male supportive staff members in a tertiary care hospital of Sri Lanka \\ Chathurie Suraweera $^{1 \star}$, Iresha Perera ${ }^{1}$, Priyanka Rupasinghe ${ }^{2}$ and Janith Galhenage ${ }^{1}$ \\ ${ }^{1}$ Professorial Psychiatry Unit, National Hospital of Sri Lanka and ${ }^{2}$ Department of Paediatric Neurology, Lady Ridgeway Hospital for Children \\ ${ }^{*}$ Corresponding author.}

doi: 10.1192/bjo.2021.778

Aims. The study describes the prevalence and associated sociodemographic variables of psychoactive substance use among male supportive staff members at a tertiary care hospital in Sri Lanka.

Method. A cross-sectional descriptive study was carried out among male supportive staff members of a tertiary care hospital in Colombo District, Sri Lanka by using a self-administered anonymous questionnaire. Participants were recruited using stratified cluster sampling in thirteen overseer divisions of the hospital. Anonymous questionnaires were collected into a sealed box and analysed using Statistical Package for Social Sciences 20. Result. The mean age of the 404 male staff members who participated in the study was $38.78(\mathrm{SD}=10.90)$ years and $71.5 \%$ were married. Among them 202 (49.1\%) were educated up to grade 6-11 and 30 of them has had encounters with law in the past. Thirty of participants had history of psychoactive substance use in the family. Alcohol was used more than once a month by $127(30.9 \%)$ and more than once a week by 19(4.6\%) individuals. Among other substances, tobacco, beetle and beedi were used by $104(25.3 \%), 78(19.0 \%)$ and $18(4.4 \%)$ respectively at least once a month. Further, 22(5.3\%), 20(4.8\%), 7(1.7\%) and 7(1.7\%) participants used Mava, Cannabis, Methamphetamine and Thool respectively at least less than once a month. Heroin, Tramadol and Morphine were used by two individuals at least less than once a month. Among substance using participants, 132 wished to cut down their habit. Most commonly identified (14.1\%) adverse consequence was financial issues secondary to psychoactive substance use. Eleven (4.5\%) staff members used the substance at hospital. Alcohol use was associated with age more than 35 years $(p=0.039)$ and history of forensic involvement $(p=0.038)$. Tobacco $(p=0.000)$, beetle $(p=0.056)$, Cannabis $(p=0.000)$ and mava $(p=0.015)$ use were significantly associated with positive forensic history. Supportive staff members' alcohol and cannabis use was associated with tobacco $(\mathrm{p}=0.000, \mathrm{p}=$ $0.000)$ and beetle use $(p=0.001, p=0.049)$. Mava use was associated with alcohol $(\mathrm{p}=0.060)$ use in addition to tobacco $(\mathrm{p}=0.020)$ and beetle use $(\mathrm{p}=0.008)$.

Binomial logistic regression revealed alcohol use and beetle use were associated with the number of children in family and above associations.

Conclusion. Commonest psychoactive substance consumed by supportive staff members were alcohol, tobacco, beetle, Cannabis and Mava in descending order of frequency. Forensic history was significantly associated with substance use. True prevalence of substance use can be higher than these values. 
Dramatic portrayal of suicide: a critical analysis of Netflix's 13 Reasons Why

Anna Sutton ${ }^{1 \star}$ and Ross Overshott ${ }^{2}$

${ }^{1}$ University of Manchester and ${ }^{2}$ Greater Manchester Mental Health NHS Foundation Trust

${ }^{*}$ Corresponding author.

doi: 10.1192/bjo.2021.779

Aims. The aims of this project were to assess how well the Netflix drama 13 Reasons Why portrayed suicide, in terms of both accuracy and safety, and to discuss the potential effect this could have on viewers.

Background. Psychiatric content within dramatic media can have measurable effects on the population, such as reinforcing stigma around mental illness. Given the show's focus on a character's suicide, the most serious effect here would be suicide contagion.

Guidelines and regulations for the portrayal of suicide in media are in place to protect those who might be vulnerable to suicide contagion.

Method. We formed our own pro-forma of 42 criteria using existing guidelines written for both news and dramatic media. These criteria were formatted into positive and negative pairs; positive being instances of guidelines being followed, negative as guidelines being broken. These were further organised into 7 categories.

Each episode of seasons 1-2 was then assessed against the criteria. Cumulative instances of guidelines being followed or broken were compared within and between seasons. Context of each instance was taken into account by the primary researcher, and we also highlighted instances of exceptional breach of these guidelines.

Result. The results showed an over-all breach of the guidelines, with no significant improvement between the seasons. Some categories of criteria, such as "asking for help" and "mental health", were portrayed well overall. Other categories, such as "blame", performed extremely badly.

The most significant breach was the graphic suicide scene at the end of the first season, which completely disregarded Samaritans' guidelines.

Conclusion. The breaching of guidelines in this show was overwhelming. In terms of severity, although there were some positive themes running through the seasons, there were also worrying instances of guidelines being completely disregarded. This led to the conclusion that the producers of the show did not take their responsibility to young, vulnerable viewers seriously regarding the dangers around portraying suicide.

Suggestions from this study are that more guidelines around suicide are needed specifically for dramatic media, and that existing guidelines should be conflated and have stronger implementation by regulators. This implementation should potentially include overseas providers such as Netflix. Ethically, a significant challenge here is maintaining balance between safety and allowing artistic licence.

\section{Psychiatric liaison referrals: a thematic analysis during peak COVID-19}

Kaj Svedberg*, William Hancox and Hugh Grant-Peterkin

City and hackney Centre for Mental Health

${ }^{\star}$ Corresponding author.

doi: 10.1192/bjo.2021.780

Aims. With the advent of the COVID-19 Pandemic the NHS long term Plan commitments of January 2019 to improve crisis care nationwide became all the more pressing. The aim of this study was to thematically investigate what mental health crisis presentations might be diverted from the Emergency department to external crisis hubs in order to reduce the COVID-19 contamination risks.

Method. All referrals made to the Homerton University Hospital (HUH) mental health liaison service were looked at between 1/3/ $20-11 / 6 / 20 \quad(n=846)$, coinciding with the first peak of the COVID-19 Pandemic.

Referral data was anonymised and sorted independently into naturally emerging thematic classes by two junior liaison doctors.

Cases that did not clearly fit any of the 14 themes generated were further looked into to determine outcome of referral and discussed to try and match to an appropriate class.

Result. 14 frequent themes for mental health crisis referrals were identified. The distribution of these ranged from most common (suicidality) to neurocognitive presentation and identified shifts in themes over the course of the pandemic peak such as increases of low mood, anxiety and intoxication requiring medical attention over the three month period.

Conclusion. Although themes for presentations may be identified in acute referrals to mental health liaison services it is problematic determining how these may be parsed safely to crisis hubs without risking overlooking cases that may require medical attention. The most common theme that was identified and remained throughout the first wave of the COVID-19 Pandemic was acute suicidal presentation. The remaining themes would require careful consideration around risk thresholds for what a service may wish to accept in devolving the emergency department liaison and balance these against future risks of repeat COVID-19 waves.

\section{Staff survey on using the new clinical risk assessment framework for teams (CRAFT) tool}

Pamela Swift ${ }^{1 \star}$, Brian Gillatt ${ }^{2}$, Emma Drysdale ${ }^{2}$, Hollie Walker ${ }^{2}$, Pamela Johnston ${ }^{2}$ and Emma Jackson ${ }^{2}$

${ }^{1}$ Royal Alexandra Hospital and ${ }^{2}$ Rowanbank Clinic

${ }^{\star}$ Corresponding author.

doi: 10.1192/bjo.2021.781

Aims. Risk assessment and management are crucial elements of clinical practice in mental health. Healthcare Improvement Scotland identified risk management as a key area for change, with risk tools identified as one necessary component. In NHS Greater Glasgow and Clyde (GG\&C) the CRAFT tool replaced the Glasgow Risk Screen (GRS) in October 2019. The CRAFT tool is a 2 page document that comprises a broad risk screen, details of historical risk events and prompts for family and carer involvement. The aim of this study was to assess staff attitudes to the CRAFT, 12 months after it had been rolled out. Looking at whether the CRAFT tool is used to inform decision making about risk in clinical settings and if patients were involved in the risk management process.

Method. An electronic staff survey was distributed to all clinical staff within NHS GG\&C Mental Health Services. Clinical staff includes the following professional groups: Medical, Nursing, Psychology, Occupational Therapists and Allied Health Professionals. Contact details were accessed via the relevant managers and surveys were sent via secure global address lists. Questions were focused around the following areas: time taken to complete/update/frequency of use/contact and ease of use, role in decision making, patient and carer involvement/knowledge, view on the impact of the CRAFT. 